EPJ Web of Conferences 32, 02010 (2012)

DOI: $10.1051 /$ epjconf/20123202010

(C) Owned by the authors, published by EDP Sciences, 2012

\title{
Applications of ECH on the DIII-D tokamak and projections for future ECH upgrades
}

\author{
R. Prater ${ }^{1}$, R.J. Buttery ${ }^{1}$, J. DeBoo ${ }^{1}$, J.R. Ferron ${ }^{1}$, A. Garofalo ${ }^{1}$, C.T. Holcomb ${ }^{2}$, G.L. Jackson ${ }^{1}$, \\ R.J. La Haye ${ }^{1}$, J.M. Lohr ${ }^{1}$, T.C. Luce ${ }^{1}$, C.C. Petty ${ }^{1}$, P.A. Politzer ${ }^{1}$, W.M. Solomon ${ }^{3}$ and F. Turco ${ }^{4}$ \\ ${ }^{1}$ General Atomics, P.O. Box 85608, San Diego, California 92186-5608, USA \\ ${ }^{2}$ Lawrence Livermore National Laboratory, Livermore, California 94550, USA \\ ${ }^{3}$ Princeton Plasma Physics Laboratory, Princeton, New Jersey 08540, USA \\ ${ }^{4}$ Columbia University, New York, New York 10027, USA
}

\begin{abstract}
Electron Cyclotron Heating and Current Drive plays an important role in the DIII-D program. In high performance discharges EC power contributes greatly to MHD stability, and this is particularly important for discharges with low rotational torque applied, as will be the case for ITER. Off-axis EC current drive also plays a key role in the actualization of steady-state scenarios by supporting the desired current profile. In order to carry out these applications at higher beta and higher field, an upgrade of the EC power to $15 \mathrm{MW}$ is needed, and the best gyrotron frequency for the DIII-D program is $117.5 \mathrm{GHz}$.
\end{abstract}

\section{Introduction}

The role of Electron Cyclotron Heating $(\mathrm{ECH})$ and Electron Cyclotron Current Drive (ECCD) in DIII-D stems from its unique and useful attributes [1], which differ substantially from the other heating methods available, primarily neutral beam injection (NBI). Unlike NBI, ECH interacts primarily with electrons, it injects no torque or particles, and its heating location in the plasma can be robustly controlled. ECCD can be driven or not, at the user's discretion, and profiles of heating and current drive can be made narrow or broad. Some applications of the existing $110 \mathrm{GHz} \mathrm{ECH} / \mathrm{ECCD}$ system on DIII-D to development and exploitation of scenarios are described.

\section{Applications of ECH/ECCD to scenarios in DIII-D}

The DIII-D program is directed in significant part to support successful operation of ITER and other future devices. One aspect of this support is carried out by generating projected scenarios for ITER in DIII-D and testing the experimental results against integrated models used to project performance, in order to improve those models. ITER differs from most present-day tokamaks in that it is expected to have relatively low applied torque compared to its high moment of inertia, and hence low toroidal rotation speeds. It will also have electron temperature equal to or larger than the ion temperature due to the plasma heating by energetic fusion products, unlike most tokamaks now that are heated by NBI. With low torque and $\mathrm{T}_{\mathrm{e}}=\mathrm{T}_{\mathrm{i}}$, it is anticipated that plasma performance at ITER-relevant 
normalized beta $\left(\beta_{\mathrm{N}}\right)$ will be different from that in more usual high torque high ion temperature discharges, and exploration of these conditions in DIII-D is underway.

The $6 \mathrm{MW}$ ECH system supplements the $20 \mathrm{MW}$ NBI heating system on DIII-D. The NBI system comprises four beamlines, each of which has two independent beam sources that supply $2.5 \mathrm{MW}$ each of neutral deuterium beams when operated at $80 \mathrm{kV}$. These beamlines were originally oriented to inject with a toroidal component in the co-current direction, but in 2006 one beamline was reversed in toroidal direction, so that it can provide injection in the opposite direction to the other beams. This modification allows operation at high NBI power but with substantially reduced toroidal torque. At total NBI power up to $10 \mathrm{MW}$ the NBI heating can be done with little net torque injected, and higher power can be applied with highly reduced torque. Scaling indicates that application of about $1 \mathrm{Nm}$ of torque in DIII-D corresponds to the anticipated $30 \mathrm{Nm}$ of torque in ITER.

One scenario being developed for ITER is the advanced inductive (AI) discharge, in which a small relatively benign $m=3 / n=2$ neoclassical tearing mode (NTM) arises spontaneously, and ECCD is used to control the $m=2 / n=1$ mode. The effect of the $3 / 2$ NTM is to broaden the current profile, creating weak central magnetic shear and avoiding sawteeth by keeping the safety factor q above 1 , while not much affecting confinement. In high torque discharges this approach works well [2], but as the applied torque is reduced toward $1 \mathrm{Nm}$ in discharges with moderately high $\beta_{\mathrm{N}}$ (above 2), a large $\mathrm{m}=2 / \mathrm{n}=1$ tearing mode typically arises, reaching an amplitude of $30 \mathrm{G}$ at the plasma edge which results in prompt toroidal locking of the plasma, with a consequent large loss of confinement and a propensity toward disruption. This behavior is shown in Figure 1, which shows two low torque advanced inductive discharges, one without $\mathrm{ECH}$ and one with $\mathrm{ECH}$ directed to drive current at $\rho=0.45$ near the $q=2$ surface.

The discharge of Figure 1 starts as a conventional high torque AI configuration, but at $3000 \mathrm{~ms}$ the beam program ramps down the $5 \mathrm{MW}$ of co-injection, Figure 1(a), and substitutes counterinjection in order to ramp down the torque, Figure 1(b). The total power throughout is in feedback mode to keep $\beta_{\mathrm{N}}$ constant at 2.8. Also at $3000 \mathrm{msec}, 3.2 \mathrm{MW}$ of EC heating and current drive is added at $\rho=0.45$. The immediate effect of the EC power is to raise the electron temperature $T_{e}$, the profiles of which are shown in Figure 2, but it also lowers the plasma toroidal rotation speed by $60 \%$, Figure 1(f), and the ion temperature $T_{i}$. The ratio $T_{e} / T_{i}$ at the plasma mid-radius, shown in Figure 1(e), increases strongly and promptly, at the application of the EC power. The confinement, characterized by the $\mathrm{H} 98 \mathrm{y}(2)$ parameter, decreases from 1.5 before the addition of EC power to a still acceptable 1.0 after, shown in Figure 1(g).

Figure 1 shows that adding substantial EC power does not decrease the NBI power needed to maintain constant plasma pressure. Raising $\mathrm{T}_{\mathrm{e}} / \mathrm{T}_{\mathrm{i}}$, as in Figure 2(a), may be detrimental to confinement, but without the EC power the discharge develops a large, rapidly growing 2/1 NTM, shown in Figure 1(c). This causes the plasma rotation to drop rapidly to zero, as shown in Figure 1(f), as the mode locks, and confinement is strongly degraded to $\mathrm{H} 98 \mathrm{y}(2)=0.5$. But when ECCD is used the low-torque high $\beta_{\mathrm{N}}$ condition is maintained to the end of the discharge. For this reason ECCD is usually applied in experiments on AI discharges.

Discharges that start with low torque, unlike that of Figure 1 but characteristic of discharges planned for ITER, invariably develop an $n=1$ mode that locks shortly after the high $\beta_{\mathrm{N}}$ is achieved, so ECH is always applied in such discharges. Even modest EC power of 0.6 to $1 \mathrm{MW}$ driving current at the $\mathrm{q}=2$ surface is sufficient to keep the $\mathrm{n}=1$ mode amplitude below $10 \mathrm{G}$, allowing sustained operation at $\beta_{\mathrm{N}}=2.5$ with zero net NBI torque without locking. 


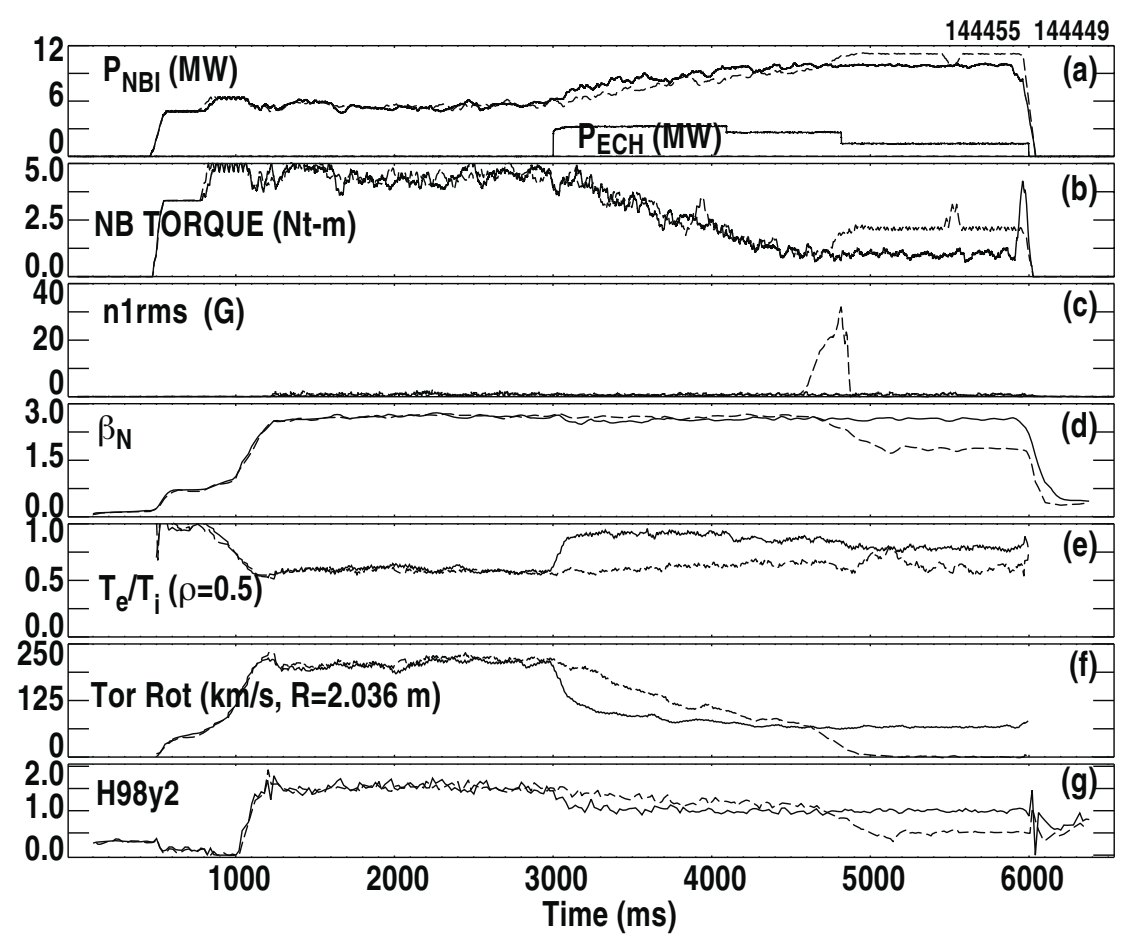

Fig. 1. A deuterium AI discharge in DIII-D with plasma current 1.05 MA, toroidal field $1.65 \mathrm{~T}$, and plasma density $4.2 \times 10^{19} \mathrm{~m}^{-3}$. (a) Neutral beam and $\mathrm{ECH}$ power; (b) injected neutral beam torque; (c) $n=1$ mode amplitude; (d) $b_{N}$ from the equilibrium reconstruction; (e) $\mathrm{T}_{\mathrm{e}} / \mathrm{T}_{\mathrm{i}}$ at the mid-minor-radius; (f) plasma rotation at the outer mid-radius; (g) confinement factor $\mathrm{H} 98 \mathrm{y}(2)$.

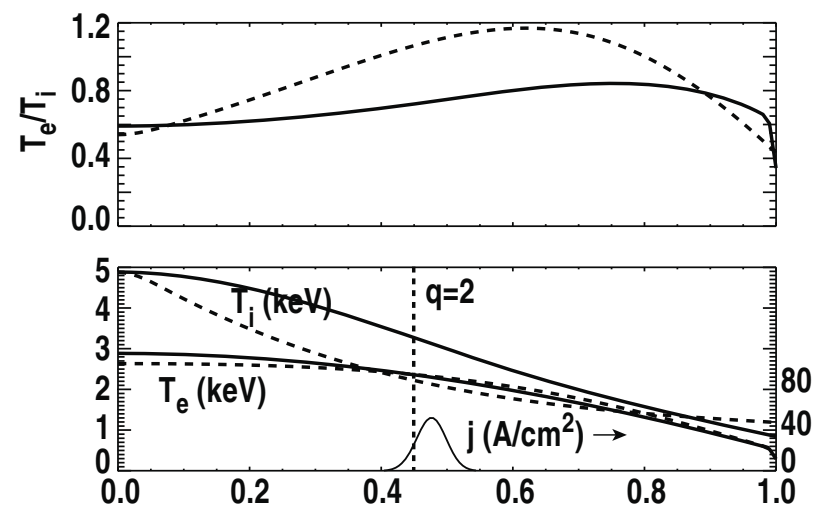

Fig. 2. For the discharge of Figure 1, (a) the radial profile of the ratio Te/Ti, and (b) the profiles of the temperatures $\mathrm{Te}$ and $\mathrm{Ti}$. The solid lines are at $2805 \mathrm{msec}$, before the EC power is applied, and the dashed lines are at $3605 \mathrm{~ms}$, with high power EC.

With the modest torque of $1 \mathrm{Nm}$, the scaled value appropriate to ITER, stable high $\beta_{\mathrm{N}}=3$ can be achieved using more central ECH. Here, $3.5 \mathrm{MW}$ of ECH spread between $\rho=0.3$ and 0.5 , where the $q=2$ surface is at $\rho=0.54$, and starting before the high $\beta_{\mathrm{N}}$ phase, is sufficient to completely avoid the $\mathrm{n}=1$ mode and maintain $\mathrm{H} 98 \mathrm{y}(2)$ above 1. This is in contrast to previous work [3] in high torque discharges that showed that the $n=1$ mode could be avoided only by keeping the ECCD placed very accurately (within 0.02 in $\rho$ ) of the $\mathrm{q}=2$ surface. Clearly the suppression mechanism in low torque 
discharges is very different. The hypothesis is that for low torque discharges the main effect of the EC power is to improve stability by affecting the classical stability parameter $\Delta^{\prime}$.

In addition to improving the MHD stability in high performance discharges, ECCD plays a major role in developing and maintaining the current profiles needed for steady state operation scenarios. This line of research is of interest for advanced steady-state scenarios in ITER but is essential for follow-on devices like a demonstration power reactor. DEMO must operate in steady-state, so full noninductive current drive is required, and in order to be economical this requires that the bootstrap fraction be large [4]. Work on DIII-D [5] has shown that discharges with the fraction of noninductive plasma current near unity require significant externally driven current in the region inside the H-mode pedestal. In DIII-D this off-axis current can be driven by a combination of neutral beam injectors that can be tilted vertically and ECCD. Calculations shown in Figure 3 using the GLF23 transport model show that steady-state operation with weak shear and q greater than 2 can be obtained in DIII-D with $9 \mathrm{MW}$ of EC source power and greater than 3 with $15 \mathrm{MW}$, when combined with off-axis NBI and central electron heating by fast waves. These fully noninductive discharges are predicted to have reactor-relevant ratio $T_{e} / T_{i}>1$ at high $\beta_{N}=3.7$. Validation of these calculations is a major objective of the DIII-D program.

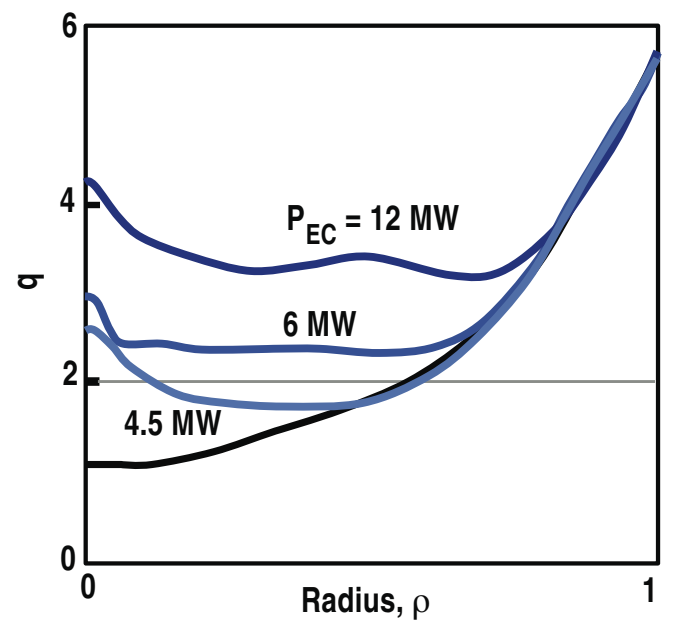

Fig. 3. Calculated profile of the safety factor q for a steady-state DIII-D discharge with onaxis neutral beams only (bottom curve), with $5 \mathrm{MW}$ of off-axis beams plus $4.5 \mathrm{MW}, 6 \mathrm{MW}$, and $12 \mathrm{MW}$ of off-axis ECCD. The toroidal field is $1.75 \mathrm{~T}$.

\section{Proposed ECH upgrade for DIII-D}

In order to fully explore in DIII-D the regime of high beta steady-state operation with $T_{e} / T_{i}>1$ and low torque, while controlling tearing modes at one or more surfaces, modelling shows that the power of the ECH system will need to be upgraded to $15 \mathrm{MW}$. This can be done most cost-effectively by adding two 1.5 MW gyrotrons and upgrading the aging $1 \mathrm{MW}$ gyrotrons in the DIII-D system with gyrotrons rated at $1.5 \mathrm{MW}$. Advances in collector geometry and materials, coupled with use of a depressed collector to reduce the power incident on the collector surface, make higher power gyrotrons more practical. In addition, improved beam tunnel design and materials increase the efficiency, and advanced designs of the mode converter reduce the stray power inside the tube while improving the coupling efficiency to waveguide.

Engineers at CPI, the gyrotron manufacturer, indicated that upgrading the power would require a different cavity mode, and hence a complete redesign of the microwave aspects of the tube. This being so, the gyrotron frequency became a parameter that could be shifted by up to $10 \%$ without 
significant effect on the cost. A study was undertaken to determine the optimum frequency for use on DIII-D. The optimization of the gyrotron frequency took into account a number of aspects:

- Operation of the high beta scenarios at toroidal fields near the maximum possible on DIII-D, $2.16 \mathrm{~T}$, is desirable. The normalized performance might be high at lower field, but the absolute performance increases with toroidal field if dimensionless parameters are held fixed.

- The efficiency of ECCD is highest when the wave-particle interaction takes place on the high field side of a flux surface, so that electron trapping is minimized.

- It is desirable to have a range of minor radius where the heating and current drive take place, for a range of toroidal field of interest.

- Excessive $3^{\text {rd }}$ harmonic absorption should be avoided.

- The maximum density accessible to EC waves is proportional to the square of the frequency. Higher density operation is frequently valuable.

These issues affect a wide range of experiment objectives for $\mathrm{ECH} / \mathrm{ECCD}$, so the motivation is to do as well as possible for the highest priority experiments - off-axis current drive and MHD stability improvement - without unduly jeopardizing the important but lower priority experiments. A true optimization can be done only for very narrowly defined objectives; instead, a range of effective operation is needed. Many of the tradeoffs can be immediately recognized from the matrix of toroidal fields and gyrotron frequencies shown in Figure 4. At $2.16 \mathrm{~T}, 110 \mathrm{GHz}$ is a poor choice because the interaction with the resonance is unavoidably on the low field side, with concomitant loss of current drive efficiency. On the other hand, $120 \mathrm{GHz}$ is a poor choice at $1.7 \mathrm{~T}$ because upshifted third harmonic absorption at the edge will be quite significant. Also, the smallest minor radius where heating can be done is $\rho=0.6$, which is too far out for many purposes.

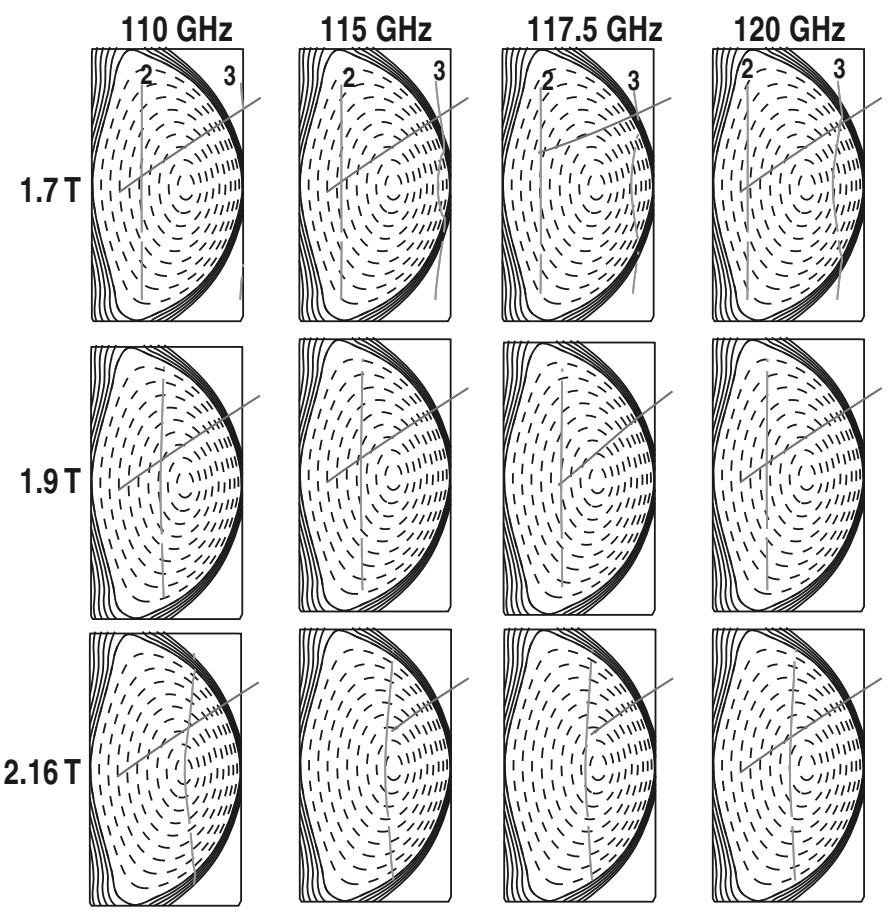

Fig. 4. DIII-D equilibria with toroidal field 1.7, 1.9, and $2.17 \mathrm{~T}$ (rows) and for gyrotron frequencies of $110,115,117.5$, and $120 \mathrm{GHz}$. The vertical lines are the $2^{\text {nd }}$ and $3^{\text {rd }}$ harmonic resonances, and the slanted lines represent typical ray bundle trajectories. The rays can be steered up and down to intersect different flux surfaces and into the paper to obtain current drive. 
An acceptable accommodation to the conditions listed above is to use $117.5 \mathrm{GHz}$ gyrotrons. Figure 5 shows contours of the minor radius and the driven current as a function of the two steering angles. Current drive from $\rho=0.3$ to 0.8 is possible, with $25 \mathrm{kA} / \mathrm{MW}$ at $\mathrm{r}=0.5$ for the conditions of this calculation. A design of the $117.5 \mathrm{GHz} 1.5 \mathrm{MW}$ gyrotron has been completed at CPI. The proposed plan to upgrade the ECH system is described in detail in [6].

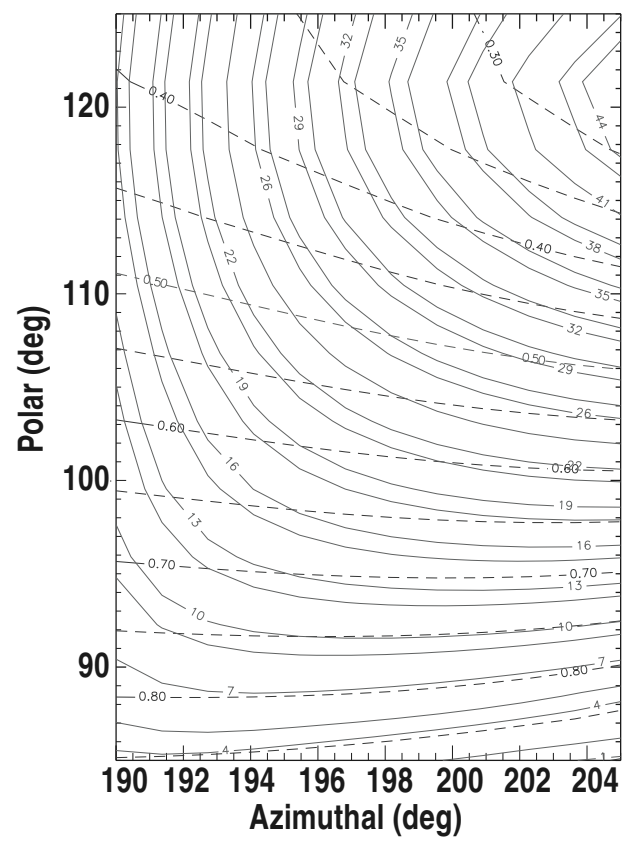

Fig. 5. Contours of the $r$ location of maximum heating (dashed lines) and contours of the magnitude of the total driven current (kA/MW), as a function of the vertical steering angle (polar angle) and toroidal steering angle (azimuthal angle). The applied frequency is 117.5 GHz, the toroidal field is $-1.9 \mathrm{~T}$, the central density is $5.5 \times 10^{19} \mathrm{~m}^{-3}$ and the central electron temperature is $7.3 \mathrm{keV}$.

\section{Acknowledgment}

This work was supported by the US Department of Energy under DE-FC02-04ER54698, DE-AC0209CH11466, and DE-FG02-04ER54761.

\section{References}

1. R. Prater, Phys. Plasmas 11, 2349 (2004)

2. M.R. Wade, et al., Fusion Sci. Tech. 48, 1199 (2005)

3. R. Prater, et al., Nucl. Fusion 47, 371 (2007)

4. T.C. Luce, Phys. Plasmas 18, 030501 (2011)

5. J.R. Ferron, et al., Nucl. Fusion 51, 063026 (2011)

6. J.M. Lohr, this workshop 